\title{
On Zone Models for Flashover
}

\author{
T.L. GRAHAM, G.M. MAKHVILADZE and J.P. ROBERTS \\ Centre for Research in Fire and Explosion Studies \\ University of Central Lancashire \\ Preston, PR1 2HE, England, UK
}

\begin{abstract}
A zone model for the development of a fire in a single compartment with one opening is studied to obtain explicit formulae for the critical conditions for occurrence of flashover and its temporal characteristics. In addition to previous studies, generalisation is presented for walls of arbitrary thermal inertia. The correlation between the model and previous models is also examined. Under reasonable assumptions all models are shown to be described by the same mathematical problem. This suggests that the formulae obtained for critical conditions and temporal characteristics can be used for any of the models observed. Results are illustrated for an experimental fire box used in many experiments.
\end{abstract}

KEYWORDS : Fire, compartment, flashover, zone modelling

\section{NOTATION}
A
$a_{i}$
$\mathrm{C}, \mathrm{C}_{\mathrm{U}}$
$\mathrm{c}_{\mathrm{D}}$
$\mathrm{c}_{\mathrm{P}}$
$\mathrm{D}$
G
$\mathrm{H}$
$\mathrm{h}$
$h, h_{u}$
$\mathrm{L}$
$\mathrm{M}_{\mathrm{X}}=\dot{m}_{\text {out }}^{\prime} c_{p}(1-D) A_{V} T_{0}$
$\mathrm{m}$
$\dot{\mathrm{m}}_{\text {out }}^{\prime}=\frac{1}{3} \mathrm{c}_{\mathrm{d}} \rho_{0} \sqrt{2 \mathrm{gH}(1-\mathrm{D})}$

surface area

dimensionless parameters $(i=0,1,2,3,4)$

constants in (5), (6)

vent discharge coefficient

specific heat capacity

fractional height of the thermal discontinuity plane

net heat gains of smoke layer

height

convective heat transfer coefficient

dimensionless parameters in (5), (6)

net heat losses from the hot zone

characteristic enthalpy flux in the Table

mass

characteristic mass outflux through opening 


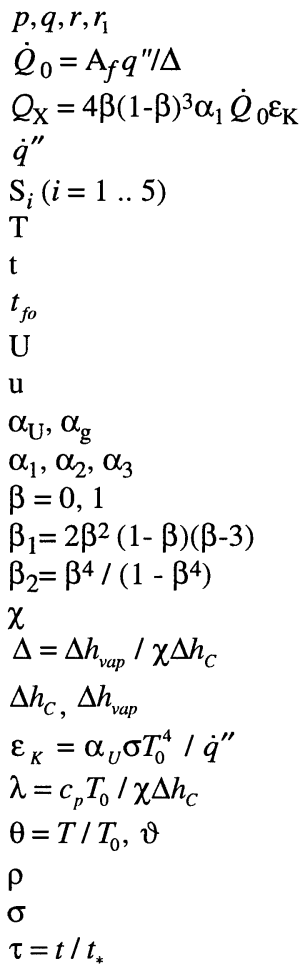

\section{SUBSCRIPTS}

* characteristic/critical value

c convection

exp expansion

g gas/smoke

$\mathrm{i}$ induction (as $\mathrm{t}_{\mathrm{i}}$ ), initial

jet initial buoyant plume

spr spread across ceiling

V vents

\author{
dimensionless parameters in (5) \\ heat release rate of fire \\ parameter in the Table \\ incident heat flux to the fuel bed from the fire \\ parameters in the Table \\ temperature \\ time \\ total time for fire growth from ignition to flashover \\ effective convective heat transfer coefficient in [1] \\ dimensionless parameter in (6) \\ emissivity of upper layer and of smoke \\ parameters in the Table \\ measure of thermal inertia of walls \\ parameter in the Table \\ parameter in the Table \\ efficiency of the combustion process \\ dimensionless heat release parameter for fire \\ heat of combustion, heat of vaporisation of solid fuel \\ characteristic radiation feedback factor \\ dimensionless constant \\ dimensionless temperature, variable of integration \\ density \\ Stefan-Boltzman constant \\ dimensionless time
}

\section{INTRODUCTION}

Flashover phenomena (the transition from a small growing fire to a fully developed fire) often accompany the development of compartmented fires. It is well understood that these phenomena are realised because of enhancement of the burning rate by factors, such as radiation from a hot smoke layer, saturation of the compartment environment by oxygen from the external atmosphere through an opening, etc. 
Theoretically, flashover is studied principally using the zone modelling approach, which gives simple and physically clear statements of the physical processes involved. Most attention has been given to flashover occurring when the burning rate of a fire is enhanced by the heat radiated from the hot smoke collected under the ceiling of a simple room with one opening. This enhancement is usually referred to as positive feedback [1].

Clear understanding of the problem was made possible when the zone modelling approach was combined with thermal explosion theory [1]. Further development of this approach has also been made by Bishop et al. [2], and Holborn et al. [3, 4] with the application of modern nonlinear dynamics.

There are still many issues to be addressed in this field. Only recently (see [5-7]) the critical conditions were stated explicitly and the temporal characteristics obtained for compartment walls of high/low thermal inertia. This work aims to further develop these relationships for flashover to include to arbitrary thermal inertia, and also to give comparison with some previous models.

\section{DESCRIPTION OF THE MODEL}

In an enclosure with one opening flashover may be described by four stages [5, 8]. Following ignition, the combustion process in the fire bed results in the formation of a 'starting' buoyant jet that impinges upon the compartment ceiling. Following this step of duration $t_{\text {jet }}$, the hot combustion products spread along the ceiling, in the time $t_{\text {spr }}$. After the time $t_{\text {jet }}+t_{\text {spr }}$ there exists a well-stirred ceiling layer of combustion products whose properties are roughly homogeneous, and the zone modelling approach can be applied. The third stage, of duration $t_{\exp }$ is the expansion and thickening of the hot layer filling downwards from ceiling height until the lower boundary of the hot layer approaches the upper level of the opening. In the fourth stage (of duration $t_{i}$ ) the mass flows through the opening rearrange. Some part of the combustion products flows out of the opening, the zone interface and neutral location exist at a level within the opening. The hot smoke and warming room boundaries radiate heat back to the fire. Radiative feedback can enhance the reaction rate so that the fire accelerates and rapidly reaches its fully developed stage. If the fire does not flashover it will burn in a quasisteady low-intensity fashion.

The full time for flashover given by $t_{f o} \approx t_{j e t}+t_{s p r}+t_{\text {exp }}+t_{i}$ has been explicitly given in a previous paper [5] where each stage was separately considered for a given, fixed fire area and for walls of large or small thermal inertia. Here we will consider the fourth stage of the development of the smoke layer assuming the initial temperature of the hot layer $\theta_{\mathrm{i}}=T_{\mathrm{i}} / T_{0}$ ( $T_{0}$ is the ambient room temperature) as was found from a heat balance consideration of the gas inside the compartment during the stage of expansion [5, 9]. Note that if the total energy released by the fire until the end of the expansion stage is much less than the total heat energy that is stored within the smoke layer then $\theta_{\mathrm{i}} \approx 1$ as was demonstrated in [5].

The model used in this paper was described in detail in [5]. A fire compartment with one opening (window) is considered under the following main assumptions: the compartment can be divided into two zones that may be represented by average temperatures; flashover takes 
place during the early development of the fire, and average density and temperature of the lower zone may be assumed to be its initial value $\left(\rho_{L} \approx \rho_{0}, T_{L} \approx T_{0}\right)$; the wall surfaces surrounding the zones can be described by two temperatures, the lower zone and wall surfaces below the thermal discontinuity are at the initial temperature; the depth of the hot layer (and accordingly the fractional height of the thermal discontinuity plane) is assumed to be a constant during the fourth stage of development leading to flashover (see $[5,9])$.

The fire area does not change appreciably and can be assumed to be constant, during the fire development the pressure inside the compartment does not significantly differ from the initial (atmospheric) pressure, i.e. the vent area is large enough. The temperature of the walls $T_{W}=T_{0}+\beta\left(T-T_{0}\right)$, where $0 \leq \beta \leq 1$ represents the thermal response of the walls $(\beta=1$ for walls of low thermal inertia and $\beta=0$ for walls of large thermal inertia). We do not account here for factors such as fuel/air exhaustion. The emissivity of the upper layer is assumed to be unity.

The governing equation of the model is of the form of a heat flux balance for the hot smoke layer which can be written in the form (see $[2,5])$ :

$m \mathrm{c}_{\mathrm{P}} \frac{d T}{d t}=G-L$

where $t$ is the time, $T$ is the smoke/hot zone temperature, $\mathrm{m}$ is the total mass in the hot layer, $c_{P}$ is the specific heat capacity. On the right hand side $G(T)$ is the net heat gain and $L(T)$ is the net heat loss from the hot zone. In (1) we have ignored the rate of increase in the enthalpy of the hot layer via mass increase, since it is usually small in relation to the total heat balance of the hot layer. Applying classical thermal explosion theory, [10] the critical conditions are given by $G=L, G^{\prime}=L^{\prime}$. Expressions for $\mathrm{G}$ and $\mathrm{L}$, and all relevant physical relationships are given in detail in [5].

For the purpose of this paper we introduce the dimensionless temperature $\theta=T / T_{0}$ and time $\tau=t / t_{*}\left(t_{*}=m c_{p} T_{0} / \dot{Q}_{0}, \dot{Q}_{0}\right.$ is the heat release rate) to yield (1) in the form [5]:

$$
\frac{d \theta}{d \tau^{\prime}}=1+a_{1}\left(\theta^{4}-1\right)-a_{2}(\theta-1)+a_{3} \theta^{3}+a_{4} \theta^{2}, \quad \theta(0)=\theta_{i}
$$

where $d \tau^{\prime}=a_{0} d \tau$ and the coefficients $a_{0}$ to $a_{4}$ are given in the Table, in the second column headed [5]. The $i$ th row of the table gives expressions for parameter $a_{i}$. For shorter notation each parameter is given in combination with some dimensionless parameters written in column 1. The four dimensionless parameters $a_{1}, a_{2}, a_{3}$, and $a_{4}$ are ratios of characteristic heat transfer rates. A linear temperature dependence $\left(T-T_{0}\right) / 2 T_{0}$ was substituted in place of $\left(T-T_{0}\right)^{3 / 2} / T T_{0}^{1 / 2}$ in the $a_{2}$ term describing the enthalpy mass outflux through the vent. This is of sufficient accuracy over the temperature range of interest. The critical conditions for flashover mentioned above in these non-dimensional variables are: 
$1+a_{1}\left(\theta_{*}^{4}-1\right)-a_{2}\left(\theta_{*}-1\right)+a_{3} \theta_{*}^{3}+a_{4} \theta_{*}^{2}=0$

$4 a_{1} \theta_{*}^{3}-a_{2}+3 a_{3} \theta_{*}^{2}+2 a_{4} \theta_{*}=0$

where $\theta_{*}$ is the critical temperature.

\section{CRITICAL TEMPERATURE}

From this formulation (4) the critical temperature is expressed in a cubic polynomial that will in general have three roots. One of these roots is always a real value (complex roots appear in conjugate pairs), and this is the critical temperature that we require. Critical temperature is an injective (ie. one-to-one) function of $\beta$. The trigonometric method [11] applied to (4) yields the critical temperature:

$\theta_{*}=h \cdot \sinh \left[\frac{1}{3} \sinh ^{-1} C\right]-r_{1}$

where $\quad h=|2 p / 3|^{1 / 2}, \quad C=q / h^{3}, \quad$ and the constants $\quad p=\left(8 a_{1} a_{4}-3 a_{3}^{2}\right) / 8 a_{1}^{2}$, $q=\left(8 a_{1}^{2} a_{2}+4 a_{1} a_{3} a_{4}-a_{3}^{3}\right) / 8 a_{1}^{3}, \quad a_{1} r=1-a_{1}+a_{2}+a_{2} r_{1}+a_{4} r_{1}^{2}-3 a_{1} r_{1}^{4}, r_{1}=a_{3} / 4 a_{1}$. Figure 1 shows the critical dimensionless temperature for the range of values of $\beta$. The example of a $0.4 \mathrm{~m}$ cubical compartment as used in [3] with an $185 \times 185 \mathrm{~mm}^{2}$ fuel tray and a $0.1 \mathrm{~m}$ opening width was used. In addition to the data in [3] the height of the discontinuity above the floor was taken to be half the room height, and the emissivity of smoke was taken to be unity.

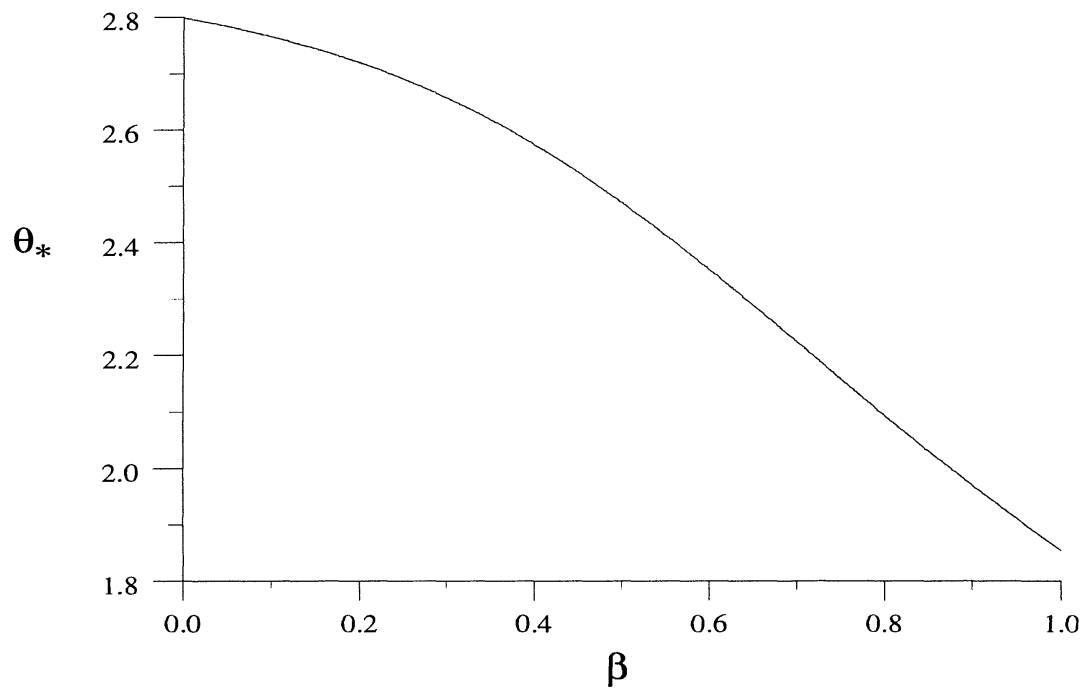

FIGURE 1. Variation of critical dimensionless temperature with thermal inertia of enclosure walls for example compartment described below 
The values of the governing parameters also vary with thermal inertia. The greater the thermal inertia of the walls, the more heat is lost to the walls in heating them and thus the greater the critical temperature for flashover.

The effect of the thermal inertia of the walls is remarkable. In this example the difference between the critical temperatures at the two extremes $\beta=0,1$ is approximately $260 \mathrm{~K}$. This shows the great importance of the heat transfer properties of the compartment walls.

\section{THE CRITICAL CONDITIONS}

A solution of the quartic polynomial (3) is possible using the radical method and gives the four roots:

$$
\begin{aligned}
& \theta_{*_{1,2}}=-\frac{(u-p)^{1 / 2}}{2} \pm \sqrt{\frac{(u-p)}{4}-\frac{q}{2(u-p)^{1 / 2}}-\frac{u}{2}}-r_{1} \\
& \theta_{* 3,4}=\frac{(u-p)^{1 / 2}}{2} \pm \sqrt{\frac{(u-p)}{4}+\frac{q}{2(u-p)^{1 / 2}}-\frac{u}{2}}-r_{1}
\end{aligned}
$$

where $u$ is given by: $u=h_{u} \cdot \cos \left[\frac{1}{3} \cos ^{-1} C_{u}\right]+p / 3$, and $h_{u}=\frac{2}{3} \sqrt{12 r+p^{2}}$, $C_{u}=4\left(\frac{2}{27} p^{3}+q^{2}-\frac{8}{3} p r\right) / h_{u}{ }^{3}$. For quasi-steady development two of the roots (6) will be real values $\theta_{*_{3}}<\theta_{*_{4}}$. If flashover does not occur then the lower of these roots is the steadystate temperature $\theta_{*_{3}}=\theta_{s}$. As any point $\left(a_{1}, a_{2}, a_{3}, a_{4}\right)$ in the quasi steady region moves towards the flashover region (for example as the fire develops and walls warm), the two real roots converge towards the critical temperature $\theta_{* 3,4} \rightarrow \theta_{*}$. Thus for a point on the critical boundary the discriminant of $\theta_{* 3}$ and $\theta_{*_{4}}$ in (6) must vanish. In addition to (5) this yields:

$\theta_{*}=\frac{(u-p)^{1 / 2}}{2}-\frac{a_{3}}{4 a_{1}}$

The critical boundary between regimes of quasi steady development and flashover must satisfy both of the conditions (3) and (4), and thus must satisfy (5) and (7). This gives:

$$
h_{u} \cdot \cos \left[\frac{1}{3} \cos ^{-1} C_{u}\right]=4 h^{4} \cdot \sinh ^{2}\left[\frac{1}{3} \sinh ^{-1} C\right]+2 p / 3
$$

For $\beta=0,1$ we have $p=0$ and the heat balance simplifies to the same form as in $[5,6]$.

The critical relationship between parameters $a_{1}, a_{2}$ plotted from (8) on Fig. 2, where we have used the same example of a $0.4 \mathrm{~m}$ cubical compartment as used in [3] for $a_{3}=0.0138 \beta^{3}(1-\beta)$ will vary continuously with $\beta$. In the two extreme cases $\beta=0,1$ the critical relationship in the plane of the parameters $a_{1}, a_{2}$ is the same [5]. In Figure 2 the two lines plotted for these cases coincide exactly. 


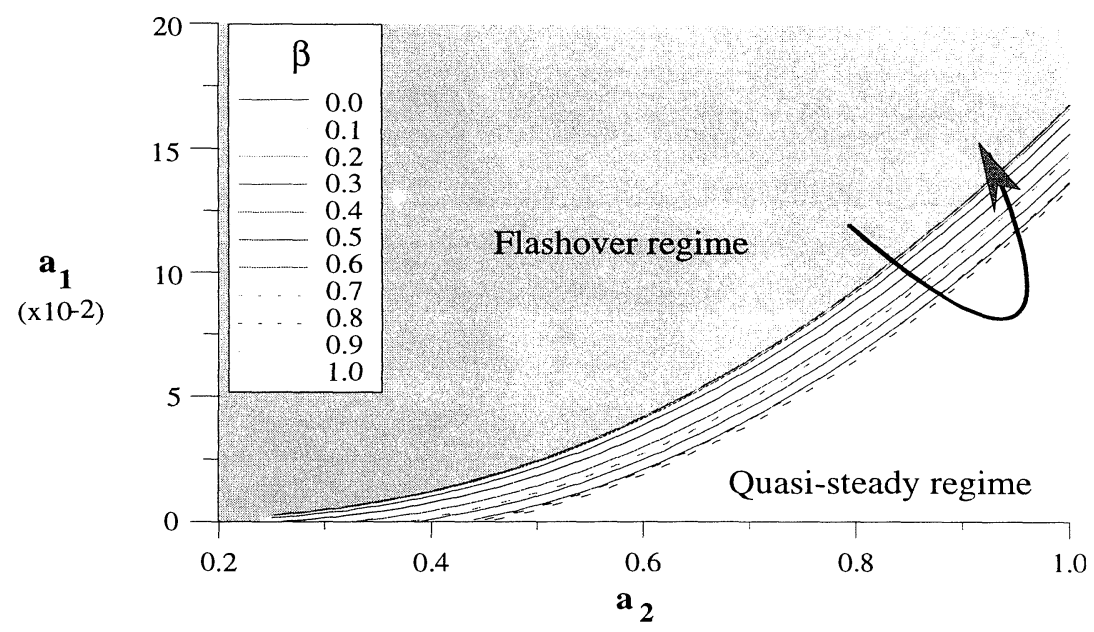

FIGURE 2. Variation of critical boundary with thermal inertia

For intermediate values the boundary curve is smoothly translated down and then back up the $a_{1}$ axis, with a maximum deviation of approximately 0.025 in $a_{1}$.

The non-monotonic dependence of the critical boundary on $\beta$ can be understood from consideration of the behaviour of the values of $a_{3}$ and $a_{4}$. The parameters $a_{3}$ and $a_{4}$ characterise radiation heat exchange with the compartment walls, arising from the binomial expansion of $T_{W}^{4}=\left[T_{0}+\beta\left(T-T_{0}\right)\right]^{4}$ at $\beta \neq 0,1$.

Physically as $\beta$ increases from 0 to 1 the heat feedback from the hot walls to the smoke layer by re-radiation increases, and the radiation losses to the walls decrease. Thus the two parameters have maxima for walls of intermediate values of thermal inertia. The greatest flashover area on Fig. 2 occurs when the product $a_{3} \cdot a_{4}$ has a maximum (at $\beta=5 / 8$ ).

\section{THE INDUCTION PERIOD}

Inverting (2) and integrating gives the temporal characteristic for the fire development in the fourth stage $c$, development:

$\tau=\int_{\theta_{i}}^{\theta} \frac{d \vartheta}{1+a_{1}\left(\vartheta^{4}-1\right)-a_{2}(\vartheta-1)+a_{3} \vartheta^{3}+a_{4} \vartheta^{2}}$

This integration may be performed by factorising the polynomial on the right hand side of (3) in terms of its four roots (6). In the limit $\theta>>1$ and for parameters $a_{1}$ to $a_{4}$ inside the flashover range of parameters this gives the induction period for development to flashover. Figure 3 shows several curves plotted by this analytic method. 


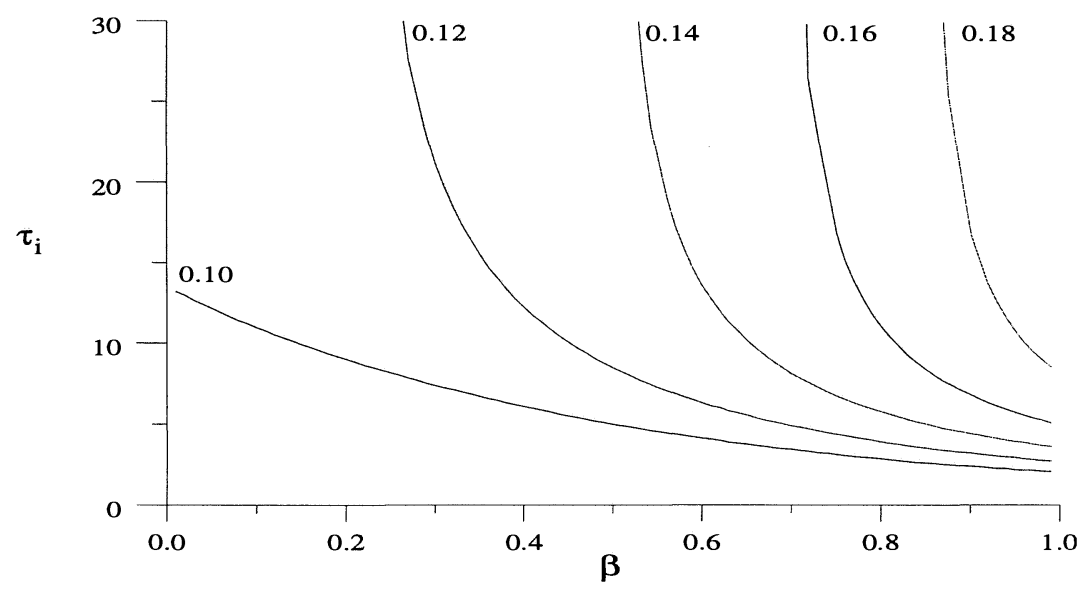

FIGURE 3. Variation of dimensionless induction period $\tau_{i}$ with thermal inertia of the walls.

Here the same example of a $0.4 \mathrm{~m}$ cubical compartment is used as before for different widths of the compartment opening, which are given next to each curve on the figure in meters.

If the thermal inertia of the walls increases (smaller $\beta$ ) then the losses to the walls increase and the induction period is longer. Furthermore for larger openings the losses are greater, and again the induction period increases. The critical opening size of the compartment for flashover decreases as thermal inertia increases.

\section{COMPARISON OF MODELS}

All zone models for early fire development in a ventilated enclosure are derived from similar thermo-physical considerations, often using similar assumptions to represent elements of the same group of phenomenon. The question arises whether or not the expressions obtained in this work in terms of the general parameters $a_{1}, a_{2}, a_{3}, a_{4}$ can be used for other models. How general are these expressions and how general is the model? Given also the proliferation of studies and developments over recent years it is increasingly useful to compare the models and indeed look at the framework for comparisons.

In this section we shall examine four models [1], [2] (see also [3]), [5] (see also [6,7]) with the developments described in this paper, [12] and their differences. We will demonstrate that each can be reduced to the same mathematical problem under reasonable assumptions, and show that the formulae and results given in [5] are general. The relationship between some earlier models has been given in [13].

For the comparison of models we must explicitly write the heat balance equation for each model in the form (1) using our notation and then introduce the dimensionless variables as described after (1). After this procedure each of the models is described by a first order nonlinear ordinary differential equation. These equations give the rate of change in upper layer temperature with time as polynomials of temperature of whole order. This representation with 
whole order polynomials is possible using our approximation for the enthalpy mass outflux through the vent, see above text after equation (2).

Consider the model presented in [1]. This is described by equation (2) with the parameters in the Table, column three headed [1]. In [1] a linear representation for radiation heat losses from the smoke layer was used. This linearisation makes it possible to eliminate the wall surface temperature $T_{W}$ from the governing equations. The effective heat transfer coefficient $U$ is introduced as a result (which will have a value of approximately $10 \mathrm{~W} / \mathrm{m}^{2} / \mathrm{K}$ ) depending implicitly on the thermal inertia of the walls. The parameters $a_{3}$ and $a_{4}$ are therefore zero. Indeed taking $\beta=0,1$ in our parameters (see the Table, column 2) gives expressions for $a_{1}, a_{2}, a_{3}, a_{4}$ close to those from [1]. This may be seen by comparing the second and third columns of the Table.

The principal difference between the parameters from our model and those in [1] is the presence of a non-linear radiation loss term making $a_{1}$ smaller than in [1]. A consequence of this is that we predict greater critical temperatures and a longer induction period when flashover occurs, and also we can conclude that flashover is slightly more likely to occur using the model in [1] because of this over estimate in $a_{1}$.

In models [2] and [12] the non-dimensional governing equation includes an extra term $-a_{5} \theta^{5}$ $\left(a_{5} \approx \lambda \varepsilon_{K}\right.$ ) arising because the enthalpy outflux through the opening $\dot{m}_{o} c_{p} T_{0}(\theta-1)$ is expressed in terms of the mass flux from the fire $\dot{m}_{f} \sim\left(\theta^{4}-1\right)$ via the mass conservation relationship $\dot{m}_{o}=\dot{m}_{a}+\dot{m}_{f}$. However since the parameter $\lambda \ll 1$ (characteristic of the heating of smoke by the fire), the parameter $a_{5}$ is small too and for many fuels may be neglected. For the data in [3] it is found that $a_{5}=1.42 \times 10^{-4}<<1$ which is small enough to be neglected. Then the process is described by equation (2).

In the model [12] the hot wall surface temperature $T_{W}$ was found by numerical solution of the heat conduction equation for the wall. The representation (2) is made possible in this case by reasonably assuming the same dependence of wall temperature on the smoke layer temperature as was used in [2,3] and [5].

The models [3] and [12] both introduce a convective heat transfer term to increase the heat flux to the fire bed $\dot{q}^{\prime \prime}$. To our mind including this convective term, in the case of a diffusion flame, requires some additional arguments.

Our model [5] includes the reasonable assumption that the density of the lower zone is close to its initial value $\left(\rho_{L} \approx \rho_{0}\right)$. This makes it possible to the determine the position of the neutral plane, and avoids an analysis of complicated situations that can arise if the interface and neutral plane are not coincident (see [13]), this issue is resolved in a different manner in [14].

The term $a_{1}$ in [1], which is responsible for the enhancement of burning rate, is the simplest reasonable expression. This form was suggested in [1] (compare the term $a_{1}$ in the second and third columns of the Table) and contains only one empirical constant (with the sense of emissivity) derived from experiments as in [3]. In the case of walls having high thermal 
inertia, $\beta=0$, this expression coincides with that in [2] if it is additionally accepted that the emissivity is one. In [14] a more complicated form for this term was suggested. This results in the appearance of extra constants, which need to be determined.

The models [2] and [12] contain different view factors and emissivities in the parameters $a_{0}, a_{1}, a_{2}, a_{3}, a_{4}$, but each of the parameters contains the same physical sense and the relationship between $a_{3}$ and $a_{4}$ is the same since the same expression for $T_{W}=T_{0}+\beta\left(T-T_{0}\right)$ was used. Comparison is clearest if the cases $\beta=0,1$ are considered, but here we give the most general expressions for the Table.

Calculations using the example data from [3] show that the models [2,3] and [12] predict greater critical temperatures and longer induction periods than our model. Furthermore our model predicts that it is easier to achieve flashover from a given room and fire geometry. However, the most general conclusion is that under reasonable assumptions all the models are described by the same mathematical problem with coefficients $a_{1}$ to $a_{4}$ given in the table. The consequence of this is that the same general formulae for critical conditions and temperature/time characteristics obtained may be applied to all four models. Furthermore, the full picture of development from ignition to flashover specified in [5] is general and can include any zone model to describe the fourth stage of the entire process..

\section{CONCLUSION}

The model of flashover in a compartment with one opening described in this work generalises results previously obtained in [5] to compartment walls of arbitrary thermal inertia. With this generalisation the analytic expressions for critical temperature and critical conditions are obtained. The time history of the system is obtained by integration.

Physically, an increase in the thermal inertia of the walls results in increased critical temperature. The boundary between the regime of quasi-steady low-intensity fire development and the regime of development to flashover varies non-monotonically in the plane of the main dimensionless parameters $\left(a_{1}, a_{2}\right)$ with the thermal inertia of the compartment walls.

The general formula obtained have a broader meaning than just within the model suggested by the authors. It is shown in the work that at least three other zonal models for flashover reduce to the same mathematical problem using reasonable assumptions.

This demonstrates that formula (5) for critical temperature, (8) for critical conditions, and also (9) for the time development of the fire to flashover can be validly used for the models examined. The parameters $a_{1}, a_{2}, a_{3}, a_{4}$ should be calculated in the way tabulated in this work.

Further verification of the model against experimental data is under way.

\section{ACKNOWLEDGEMENTS}

The authors would like to express their gratitude to the Engineering and Physical Sciences Research Council, GR/J85035, for their promotion of this study. 


\section{REFERENCES}

1. Thomas, P.H., Bullen, M.L., Quintiere, J.G., McCaffrey, B.J., "Flashover and instabilities in fire behaviour", Combustion and Flame, 38: pp. 159-171, 1980.

2. Bishop, S.R., Holborn, P.G., Beard, A.N., Drysdale, D.D., "Nonlinear dynamics of flashover in compartment fires", Fire Safety Journal, 21: pp. 11-45, 1993.

3. Holborn, P.G., Bishop, S.R., Drysdale, D.D., Beard, A.N., "Experimental and theoretical models of flashover", Fire Safety Journal, 21: pp. 257-266, 1993.

4. Beard, A.N., Drysdale, D.D., Holborn, P.G., Bishop, S.R., "A model of instability and flashover", Journal of Applied Fire Science, 4: 1, pp. 3-16, 1994-5.

5. Graham, T.L., Makhviladze, G.M., Roberts, J.R., "On the theory of flashover development”, Fire Safety Journal, 25: 3, pp. 229-259, 1995.

6. Graham, T.L., Makhviladze, G.M., Roberts, J.R., "Critical conditions for flashover", in Fire Engineering and Emergency Planning; Research and Applications,

E. \& F. Spon, London, ed. R. Barham, pp. 18-26, 1995.

7. Graham, T.L., Makhviladze, G.M., Roberts, J.R., "On flashover in compartment fires", in Fire Safety by Design, Northern Branch Conference, Institute of Fire Engineers, Sunderland, 3, pp. 157-167, 1995.

8. Thomas, P.H., "The growth of fire-ignition to full involvement", in Combustion Fundamentals of Fire, Editor G. Cox, Academic Press, 5, pp. 273-328, 1995.

9. Zukoski, E.E., "Development of a stratified ceiling layer in the early stages of a closed room fire", Fire Materials, 2: pp. 54-62, 1978.

10. Zeldovich, Ya.B., Barenblatt, G.I., Librovich, V.B., Makhviladze, G.M.,."The mathematical theory of combustion and explosions", Plenum Press, New York, USA, 1985.

11. Birkhoff, G., Mac Lane, S., A survey of modern algebra, Macmillan, New York, 1965.

12. Takeda, H., "Transient model of early stages in compartment fires", in Mathematical modelling of fires, Editor J.R. Mehaffey, ASTM, Philadelphia, pp. 21-34, 1987.

13. Thomas, P.H., "On a relationship between certain zone models of two layer and uniform compartment fires", Fire Science and Technology, 4: 2, pp. 65-74, 1984.

14. Beard, A.N., Drysdale, D.D., Holborn, P.G., Bishop, S.R., "A non-linear model of flashover", Fire Science and Technology, 12: pp. 11-27, 1992. 


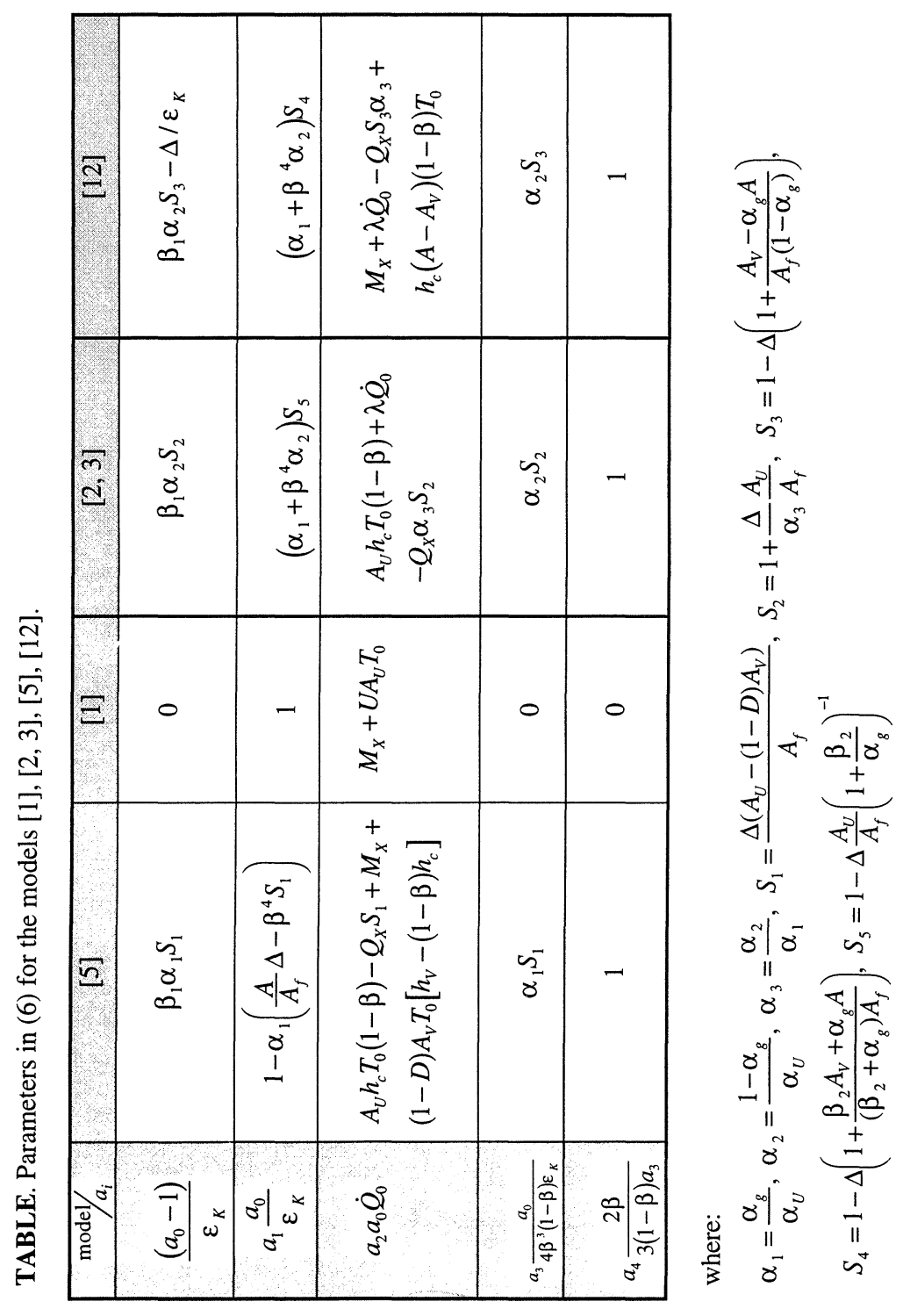

